\title{
Evaluation of pretreatment serum interleukin-6 and tumour necrosis factor alpha as a potential biomarker for recurrence in patients with oral squamous cell carcinoma
}

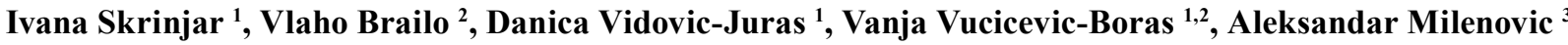 \\ ${ }^{1}$ Department of Oral Medicine, Clinical Hospital Center Zagreb, Gunduliceva 5, 10000 Zagreb, Croatia \\ ${ }^{2}$ Department of Oral Medicine, School of Dental Medicine, University of Zagreb, Gunduliceva 5, 10000 Zagreb, Croatia \\ ${ }^{3}$ Department of Maxillofacial Surgery, University Hospital Dubrava Zagreb, Av. Gojka Suska 6, 10000 Zagreb, Croatia
}

Correspondence:

Department of Oral Medicine

Clinical Hospital Center Zagreb

Gunduliceva 5

10000 Zagreb, Croatia

skrinjar.ivana@gmail.com

\begin{abstract}
Skrinjar I, Brailo V, Vidovic-Juras D, Vucicevic-Boras V, Milenovic A. Evaluation of pretreatment serum interleukin-6 and tumor necrosis factor alpha as a potential biomarker for recurrence in patients with oral squamous cell carcinoma. Med Oral Patol Oral Cir Bucal. 2015 Jul 1;20 (4):e402-7. http://www.medicinaoral.com/medoralfree01/v20i4/medoralv20i4p402.pdf
\end{abstract}

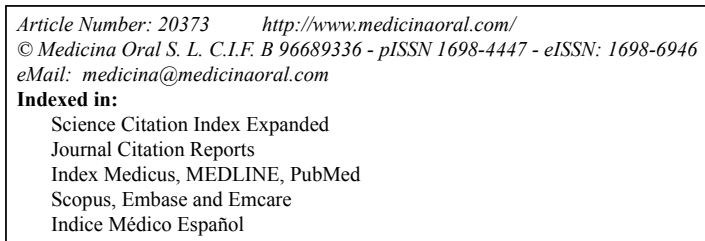

\begin{abstract}
Background: Oral squamous cell carcinoma (OSCC) constitutes 3 percent of all cancers with predominant occurrence in middle aged and elderly males. Tumour recurrence worsens disease prognosis and decreases quality of life in patients with OSCC. Proinflammatory cytokines such as interleukin-6 (IL-6) and tumour necrosis factor alpha $(\mathrm{TNF}-\alpha)$ have been suggested to play a certain role in variety of tumours. The aim of this study was to investigate the relationship of pretreatment serum IL- 6 and TNF- $\alpha$ levels on tumour recurrence in patients with OSCC in order to identify potential biomarkers for the early detection of disease recurrence.

Material and Methods: The patients with newly diagnosed OSCC were treated and followed from the first visit from November 2006 until January 2008. Serum IL-6 and TNF- $\alpha$ concentrations were measured. The records of the patients were re-examined in July 2012 and data were recorded about cancer characteristics and tumour recurrence. Disease free survival was analyzed by Kaplan-Meier survival curves, log rank test and Cox proportional hazards regression.

Results: Serum IL-6 was shown as an independent risk factor for tumour recurrence.

Conclusions: Pretreatment serum IL-6 concentration may be a useful biomarker for identification of OSCC patients with increased risk of the disease recurrence.
\end{abstract}

Key words: Serum IL-6, serum TNF- $\alpha$, oral cancer, recurrence. 


\section{Introduction}

Oral squamous cell carcinoma (OSCC) accounts for $95 \%$ of all oral cavity and oropharyngeal cancers (1). Despite the improvement in surgical and radiation therapy, 5-year survival rate has not significantly improved. The stage of the tumour at the time of diagnosis is important predictor of survival. Tumour recurrence significantly worsens disease prognosis and decreases quality of life in patients with OSCC.

It has been shown that inflammation plays a key role in different stages of carcinogenesis (2). Serum concentrations of cytokines can be attributed to the secretion from tumour cells as well to systemic immune response to the tumour growth (3).

Proinflammatory cytokines such as interleukin-6 (IL-6) and tumour necrosis factor alpha (TNF- $\alpha$ ) have been suggested to play a certain role in the variety of tumours. The increased levels of serum IL-6 in patients with head and neck squamous cell carcinoma (HNSCC) compared to the healthy controls have been found in previous reports (4-6). Serum IL-6 concentration might correlate with the stage of tumour proliferation in patients with HNSCC (7) and can be useful in the detection of metastases (8).

It has been shown that TNF- $\alpha$ could act as endogenous promotor of tumour via activation of NF-kB transcriptional factor (9). Different tumour cells produce TNF- $\alpha$, including ovarian cancer and breast cancer (2). Recent study has shown that high levels of plasma TNF- $\alpha$ and $\mathrm{C}$-reactive protein affect survival in patients with $\mathrm{HN}-$ SCC (10).

The aim of this study was to investigate the correlation of serum IL- 6 and TNF- $\alpha$ levels on tumour recurrence in patients with OSCC in order to identify potential biomarkers for early detection of disease recurrence.

\section{Material and Methods}

This was a prospective study of patients with newly diagnosed OSCC. The patients were treated and followed from the first visit (November 2006 - January 2008) until June 2012 at the Department of Maxillofacial Surgery University Hospital Dubrava Zagreb, Croatia. The study included 36 patients ( 30 man and 6 women) with histologically confirmed OSCC, mean age 59 (range 37-78) and 31 healthy volunteer blood donors (19 man and 12 women) with healthy oral mucosa, mean age 58 (range 50-78). Cancer stage was determined by use of TNM classification according to American Joint Committee on Cancer. Exclusion criteria for OSCC patients was assigned diagnosis of autoimmune disease.

Prior to the study, all the participants signed informed consent and the study was performed in accordance with the Declaration of Helsinki. The study was approved by the Ethics Committee of the School of Dental Medicine University of Zagreb and financed by the
Croatian Ministry of Science and Technology, project number 065-0650445-0485.

The recruitment of the patients began in November 2006 when blood samples for IL- 6 and TNF- $\alpha$ analysis were collected. Notations were made about age, sex, smoking status and cancer site. Cancer site was divided into anterior and posterior regions of the mouth. Periodontal status was determined by Community Periodontal Index of Treatment Needs (CPITN) (11). The records were again re-examined in July 2012 and data were recorded again about cancer stage, $\mathrm{N}$ classification, type of treatment and local recurrence, regional recurrence and/or metastatic disease. The type of treatment was dichotomized as surgery with/without radiotherapy versus chemoradiotherapy. The patients were censored as having a recurrence or not at their last annual chart review. Time until recurrence was noticed as disease free survival.

- Blood collection and cytokines analysis

Blood samples were collected between 8 and 10 A.M. with standard method from the antecubital veins. In patients with OSCC blood was collected before any kind of treatment. Samples were stored at room temperature for 90 minutes and then centrifuged for 10 minutes (ROTINA 35, Hettich, Germany). Sera were separated with Pasteur pipette and frozen at $-70^{\circ} \mathrm{C}$ until analyzed.

Concentration of IL-6 and TNF- $\alpha$ were analyzed by chemiluminiscent enzyme-linked immune assay (ELISA).

For each cytokine $300 \mu \mathrm{L}$ of sera was put into vial and transferred into container with marbles on which primary antibodies (IMMULITE, Siemens, Germany) specific for certain cytokine were adsorbed. After incubation and washing, secondary antibody labeled with enzyme (IMMULITE, Siemens, Germany) was added. After washing unbind secondary antibody, luminogenous substrate (adamantyl-1,2-dioxetane-phosphate) was added. Degradation of luminogenous substrate results in emission of light which is measured by luminometer. Concentrations of cytokines were written by device in $\mathrm{pg} / \mathrm{ml}$. The lower limit of detection for IL-6 was $2 \mathrm{pg} / \mathrm{ml}$ and for TNF- $\alpha 4 \mathrm{pg} / \mathrm{ml}$. The highest limit of detection for both cytokines was $1000 \mathrm{pg} / \mathrm{ml}$. If the concentrations were not detectable value of $0.001 \mathrm{pg} / \mathrm{ml}$ was recorded.

Elevated levels for each cytokine were defined as higher levels than the 95 . percentile of a value in the control subjects (12).

- Statistical Analysis

Statistical analysis was performed by MedCalc 13.0.0. statistical programme (Ostend, Belgium). The Fisher exact test was used to compare cytokine concentrations between groups. The Mann-Whitney U test and Kruskal-Wallis test were used to determine the association of concentration of IL- 6 and TNF- $\alpha$ with control variables. 
Disease free survival was analyzed by Kaplan-Meier survival curves, log rank test and Cox proportional hazards regression. $P$-value $<0.05$ was considered significant in all analyses.

\section{Results}

There were no significant differences regarding age and sex between the patients and the controls.

The elevated level of serum IL- 6 was defined as $>6 \mathrm{pg} / \mathrm{ml}$ and elevated level of serum TNF- $\alpha$ was defined as $>28.6$ $\mathrm{pg} / \mathrm{ml}$ according to previous study described earlier (12). The patients with OSCC had higher concentration of serum IL-6 than the controls (Fisher exact test, $p=0.006$ ). No difference was found in the concentration of serum TNF- $\alpha$ between the patients and the controls (Table 1). Table 2 shows clinical characteristic of the disease. Most of the patients had tumour stage 3 or stage 4, and only one patient had distant metastases in the time of the diagnosis.

There were no differences between concentrations of serum IL- 6 and TNF- $\alpha$ according to the age, cancer site, cancer stage and $\mathrm{N}$ classification (Table 3 ). It was

Table 1. Serum IL-6 and TNF- $\alpha$ in oral cancer patients and control subjects.

\begin{tabular}{|c|c|c|}
\hline Serum IL-6 & OSCC patients & Controls \\
\hline$<=6 \mathrm{pg} / \mathrm{ml}$ & 28 & 31 \\
\hline$>6 \mathrm{pg} / \mathrm{ml}$ & 8 & 0 \\
\hline$p^{\mathrm{a}}$ & $\mathbf{0 . 0 0 6}$ & \\
\hline Serum TNF- $\alpha$ & & \\
\hline$<=28,6 \mathrm{pg} / \mathrm{ml}$ & 36 & 30 \\
\hline$>28,6 \mathrm{pg} / \mathrm{ml}$ & 0 & 1 \\
\hline$p^{\mathrm{a}}$ & 0.463 & \\
\hline
\end{tabular}

* Fisher exact test

Table 2. Clinical characteristics of the disease.

\begin{tabular}{|l|c|c|}
\hline \multirow{4}{*}{ Tumor localization } & N & N \\
\cline { 2 - 3 } & $\begin{array}{c}\text { Front parts of the } \\
\text { oral cavity }\end{array}$ & 11 \\
\cline { 2 - 3 } & $\begin{array}{c}\text { Back parts of the } \\
\text { oral cavity }\end{array}$ & 21 \\
\cline { 2 - 3 } & $\begin{array}{c}\text { Front and back parts } \\
\text { of the oral cavity }\end{array}$ & 4 \\
\hline \multirow{4}{*}{ Tumor size } & $\mathrm{T} 1$ & 4 \\
\cline { 2 - 3 } & $\mathrm{T} 2$ & 8 \\
\cline { 2 - 3 } & $\mathrm{T} 3$ & 12 \\
\hline \multirow{4}{*}{ N classification* } & $\mathrm{T} 4$ & 12 \\
\cline { 2 - 3 } & $\mathrm{N} 0$ & 17 \\
\cline { 2 - 3 } & $\mathrm{N} 1$ & 5 \\
\cline { 2 - 3 } Distant metastases & $\mathrm{N} 2$ & 12 \\
\cline { 2 - 3 } & $\mathrm{N} 3$ & 1 \\
\hline \multirow{4}{*}{ Tumor stage } & Yes & 1 \\
\cline { 2 - 3 } & No & 35 \\
\cline { 2 - 3 } & Stage 1 & 3 \\
\cline { 2 - 3 } & Stage 2 & 6 \\
\hline
\end{tabular}

* for one patient $\mathrm{N}$ classification is not known. $\mathrm{N}=$ number of patients.
Table 3. Serum IL-6 and TNF- $\alpha$ and contol variables.

\begin{tabular}{|l|c|c|}
\hline & $\begin{array}{c}\text { Serum IL-6 } \\
\text { (median(range), } \\
\mathbf{p g} / \mathbf{m l}\end{array}$ & $\begin{array}{c}\text { Serum TNF- } \boldsymbol{\alpha} \\
\text { (median(range), pg/ml }\end{array}$ \\
\hline Age & $2(0.001-12)$ & $7(4-17)$ \\
$<59$ years & $3(0.001-14)$ & $7(0.001-22)$ \\
$>59$ years & 0.236 & 0.972 \\
$p^{\text {a }}$ & & $7(0.001-22)$ \\
\hline Sex & $3(0.001-14)$ & $5(0.001-8)$ \\
Male & $1(0.001-2)$ & $\mathbf{0 . 0 2}$ \\
Female & $\mathbf{0 . 0 3}$ & $7.5(0.001-22)$ \\
$p^{\mathrm{a}}$ & $3(0.001-14)$ & $5(0.001-10)$ \\
\hline Smokers & $1(0.001-7)$ & $\mathbf{0 . 0 4}$ \\
Non-smokers & 0.17 & $7(4-10)$ \\
$p^{\mathrm{a}}$ & & $6(0.001-22)$ \\
\hline Cancer site & $3(0.001-12)$ & $8(6-13)$ \\
Front regions & $2(0.001-14)$ & 0.641 \\
Back regions & $0.001(0.001-4)$ & \\
Both regions & 0.206 & $7(4-11)$ \\
$p^{\mathrm{b}}$ & $3(2-12)$ & $7(0.001-22)$ \\
\hline Cancer stage & $2(0.001-14)$ & 0.485 \\
S1+S2 & 0.202 & $5(0.001-15)$ \\
S3+S4 & $2(0.001-14)$ & $7(7-22)$ \\
$p^{\mathrm{a}}$ & $2.5(0.001-12)$ & 0.288 \\
\hline N classification & 0.776 & \\
N0 & & \\
N1+N2+N3 & & \\
$P^{\mathrm{a}}$ & & \\
\hline
\end{tabular}

${ }^{a}$ Mann-Whitney U test

${ }^{\mathrm{b}}$ Kruskal-Wallis test

shown that men have significantly higher serum IL-6 and TNF- $\alpha$ levels when compared with the women ( $p=0.03 ; p=0.02$ respectively) and that smokers have significantly higher serum TNF- $\alpha$ levels in comparison to the non-smokers $(p=0.04)$. Serum IL-6 concentrations did not differ between smokers and non-smokers. The results show that patients who had distant metastases were younger (Fig. 1). Univariate analysis of tumour recurrence. Two-year recurrence rate was fourty-four percent.

Univariate analysis by Kaplan-Meier survival curves and log rank test failed to show significance between serum IL- 6 or TNF- $\alpha$ and disease free survival. Other factors including age, sex, smoking status, cancer site, cancer stage and $\mathrm{N}$ classification also did not correlate significantly with disease free survival. The treatment type was shown as an independent risk factor for recurrence (Fig. 2). The patients who underwent surgery with/without radiotherapy had median disease free survival of 1080 days when compared to the patients who solely underwent chemoradiotherapy without surgery, and who had median disease free survival of 180 days. Multivariate analysis of tumour recurrence All variables were analyzed by multivariate analysis to determine their significance when cofactors were con- 


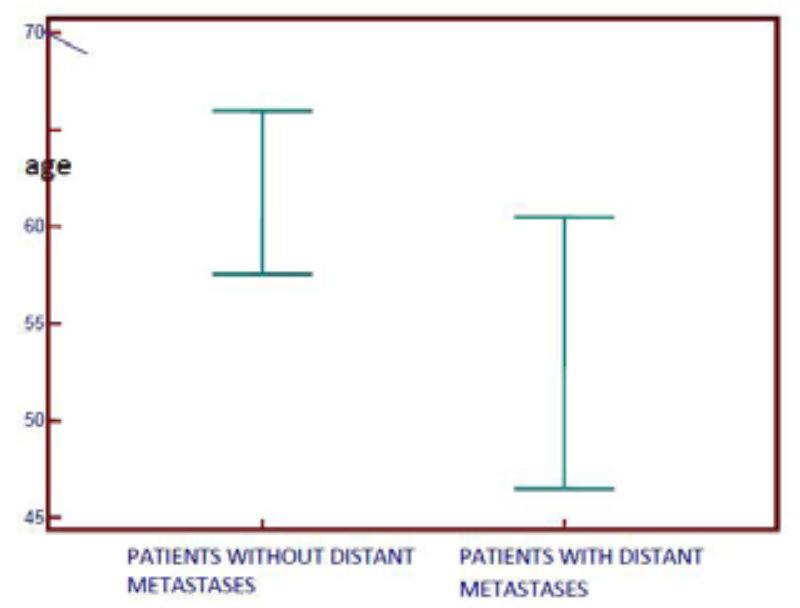

Fig. 1. Distant metastases according to age in patients with oral squamous cell carcinoma. The median age of patients with metastases was 53, the median age of patients without metastases was 61 .

trolled as was recommended in the previous study of Sun et al. (13).

For the purpose of multivariate analysis serum IL-6 and TNF- $\alpha$ was log transformed to eliminate the effect of extreme values.

The results of multivariate Cox proportional hazards models for recurrence are shown in table 4. Serum IL-6, cancer site and type of therapy were significant risk factors for tumour recurrence. Higher pretreatment serum levels of IL-6 were significantly associated with disease recurrence (HR=1.57; 95\% CI, 1.06-2.33, $p=0.03)$. The patients with tumours located in the anterior and posterior regions of the mouth had higher risk for recurrence $(\mathrm{HR}=10.59 ; 95 \% \mathrm{CI}, 2.04 .-55.06 ; p=0.005)$. The treatment type was also shown as independent factor of recurrence as was in univariate analysis $(\mathrm{HR}=24.83$; 95\% CI, 4.18-147.40, $p<0.001)$. Serum TNF- $\alpha$, age, sex, smoking status, cancer stage and $\mathrm{N}$ classification were not significantly associated with the tumour recurrence.

Table 4. Cox proportional hazards regression models for recurrence events.

\begin{tabular}{|l|c|c|c|}
\hline Significant variables & $\begin{array}{c}\text { Hazard } \\
\text { ratio }\end{array}$ & 95\% CI & $\boldsymbol{p}$ \\
\hline Log serum IL-6 & 1.57 & $\begin{array}{c}1.06- \\
2.33\end{array}$ & 0.03 \\
\hline Cancer site & 10.59 & $\begin{array}{l}2.04 .- \\
55.06\end{array}$ & 0.005 \\
$\begin{array}{l}\text { Both regions vs anterior and } \\
\text { vs posterior regions }\end{array}$ & 24.83 & $\begin{array}{c}4.18- \\
147.40\end{array}$ & $<0.001$ \\
\hline $\begin{array}{l}\text { Type of therapy } \\
\text { Surgery with/without } \\
\text { radiotherapy vs } \\
\text { chemoradiotherapy }\end{array}$ & & \\
\hline
\end{tabular}

For IL-6 the hazard ratio is for a unit increase in log serum IL-6.

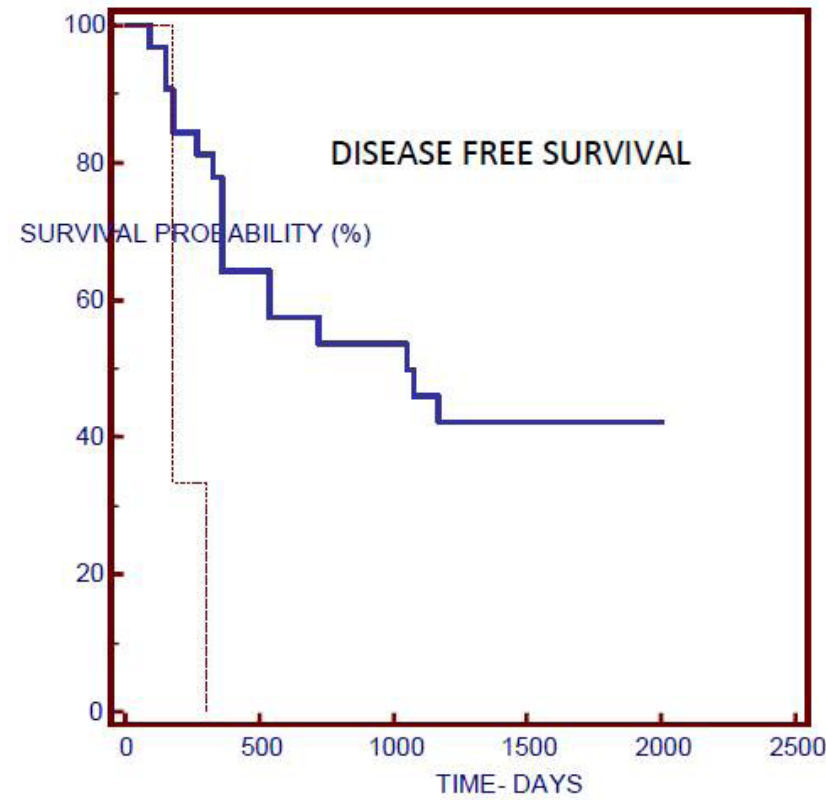

Fig. 2. A Kaplan-Meier plot of treatment type.

\section{TREATMENT TYPE \\ SURGERY WITH/WITHOUT RADIOTHERAPY CHEMORADIOTHERAPY}




\section{Discussion}

Our results have shown that pretreatment serum IL-6 concentration may be a useful marker for identification of OSCC patients with increased risk for the disease recurrence. Early diagnosis of locoregional or metastatic disease plays an important role in the prognosis of the patients with oral cancer (14-16). In this study, serum concentration of IL-6 was higher in oral cancer patients when compared to the controls which was also confirmed in other studies $(4,6,8,17)$ Furthermore, the results of this study show that serum IL-6 was an independent risk factor for the disease recurrence.

Elevated serum IL-6 concentrations indicate that systemic inflammatory response is involved in carcinogenesis. Tumour growth and invasion in the surrounding tissues induces inflammation while tumour necrosis and hypoxia may activate inflammatory response. Moreover, tumour cells themselves may increase production of inflammatory cytokines such as IL-6 (18). Those cytokines react with immune and vascular system and stimulate tumour cells growth, tumour invasion and metastases (19). Some authors $(10,20,21)$ did not report difference between serum IL-6 concentrations in patients with OSCC and controls. In the sudy of Andersson et al. (10) patients with head and neck squamous cell carcinoma were not age and sex matched with the healthy controls as it was in our study. The study of Vucicevic Boras et al. (21) had differences in age between control group (mean age 25) and patients with OSCC (mean age 55). The study of Czerninski et al. (20) included potentially premalignant disorders (oral dysplasia or oral lichen planus) and six healthy volunteers as a control against only healty volunteers in our study. Those differences in methodologies could explain different results. The results of this and other studies $(5,7,22)$ indicate that serum IL-6 is elevated in OSCC patients and may be potential marker for tumour recurrence. The patients with elevated serum IL-6 concentration may benefit from more frequent recall visits for the purpose of early diagnosis of tumour recurrence.

Although our results have shown that serum IL-6 concentration was higher in men than women, sex was not significant risk factor for tumour recurrence as was shown by other authors $(14,22)$. Although sex did not have impact on tumour recurrence, both serum IL-6 and TNF- $\alpha$ were higher in men. Some authors have shown higher serum IL- 6 and TNF- $\alpha$ levels in healthy smokers when compared to the healthy non-smokers and higher TNF- $\alpha$ levels in patients with periodontitis in comparison to the healthy controls (23). The results of this study have shown that men did not smoke significantly more than women (data not shown). There were no significant differences regarding CPITN between men and women, as well as no significant difference in CPITN between OSCC patients and healthy controls (data not shown).
Therefore, we can exclude smoking and periodontal disease as a potential causes of differences between IL- 6 and TNF- $\alpha$ serum concentration between men and women. Elevated levels of IL- 6 and TNF- $\alpha$ in men compared to women could probably be explained by the fact that we had unequal sample size regarding men and women. This result has to be confirmed or rejected in the future study with approximate equal percent of both genders.

Tumour recurrence was not dependent on the participants age which was also confirmed by others $(14,24)$. Nevertheless, patients who had distant metastases were significantly younger which confirms the fact that oral cancer is more aggressive in younger people (25).

Cancer site was not associated with serum concentrations of IL- 6 and TNF- $\alpha$. However cancer site was shown as a significant risk factor for tumour recurrence. Tumours located in the anterior and posterior regions of the oral cavity had worse prognosis in comparison to those located in the anterior or posterior regions only. There were four patients within this group and all of them had tumours which involved floor of the mouth and the base of the tongue. This finding indicates that tumours located in those regions are more aggressive which could be explained by different lymphatic drainage of the anterior and posterior regions of the mouth (26). Generally, lymph from the posterior regions drains to lower cervical lymph nodes and involvement of those nodes could be associated with poor prognosis in OSCC patients.

The treatment type was shown as an independent risk factor for tumour recurrence. The patients who underwent chemoradiotherapy only had worse prognosis in comparison to the patients who underwent surgery with/without radiotherapy. Cancer stage, on the other hand was not associated with tumour recurrence. Even though the treatment is based on the stage of the disease, different treatment modalities can be applied within the same stage based on factors like tumour site, capsule perforation, patient's general state etc. Three patients treated with chemoraditherapy had tumours localized in the posterior regions of oral cavity which could have contributed to the outcome. Given the fact that there were only three patients in this group, this result should be taken with caution.

Serum TNF- $\alpha$ concentration did not differ significantly between OSCC patients and healthy controls as was shown by other authors $(6,27)$ and was not shown to be a significant risk factor for tumour recurrence. In this study, smokers had higher serum TNF- $\alpha$ concentrations in comparison to the non-smokers which indicates systemic inflammatory response to the ingredients in cigarette smoke as it was shown previously (23).

In conclusion, pretreatment serum IL-6 concentration may be a useful biomarker for early detection of recur- 
rence in patients with OSCC. Patients with elevated pretreatment serum IL-6 levels should therefore be recalled more frequently.

Further prospective studies involving greater number of OSCC patients are needed to confirm these findings.

\section{References}

1. Ramos M, Benavente S, Giralt J. Management of squamous cell carcinoma of the head and neck: updated European tretment recommendations. Expert Rev Anticancer Ther. 2010;10:339-44.

2. Aggarwal BB, Shishodia S, Sandur SK, Pandey MK, Sethi G. Inflammation and cancer: how hot is the link? Biochem Pharmacol. 2006;72:1605-21.

3. Osman TA, Costea DE, Johannessen AC. The use of salivary cytokines as a screening tool for oral squamous cell carcinoma: A review of the literature. J Oral Maxillofac Pathol. 2012;16:256-61.

4. Druzgal CH, Chen Z, Yeh NT, Thomas GR, Ondrey FG, Duffey DC, et al. A pilot study of longitudinal serum cytokine and angiogenesis factor levels as markers of therapeutic response and survival in patients with head and neck squamous cell carcinoma. Head Neck. 2005;27:771-84.

5. St John MA, Li Y, Zhou X, Denny P, Ho CM. Montemagno C, et al. Interleukin 6 and interleukin 8 as a potential biomarkers for oral cavity and oropharyngeal squamous cell carcinoma. Arch Otolaryngol Head Neck Surg. 2004;130:929-35.

6. Hoffmann TK, Sonkoly E, Homey B, Scheckenbach K, Gwosdz C, Bas M, et al. Aberrant cytokine expression in serum of patients with adenoid cystic carcinoma and squamous cell carcinoma of the head and neck. Head Neck. 2007;29:472-8.

7. Mojtahedi Z, Khademi B, Hashemo SB, Bagher SM, Ghasemi MA, Fattahi MJ, et al. Serum Interleukine-6 Concentration, But Not Interleukine-18 is Associated with Head and Neck Squamous Cell Carcinoma Progression. Pathol Oncol Res. 2011;17:7-10.

8. Riedel F, Zaiss I, Herzog D, Götte K, Naim R, Hörmann K. Serum levels of interleukin-6 in patients with primary head and neck squamous cell carcinoma. Anticancer Res. 2005;25:2761-5.

9. Aggarwal BB, Gupta SC, Kim JH. Historical perspectives on tumor necrosis factor and its superfamily: 25 years later, a golden journey. Blood. 2012;119:651-65.

10. Andersson BÄ, Lewin F, Lundgren J, Nilsson M, Rutquist LE, Löfgren S, et al. Plasma tumor necrosis factor- $\alpha$ and C-reactive protein as biomarker for survival in head and neck squamous cell carcinoma. J Cancer Res Clin Oncol. 2014;140:515-9.

11. Ainamo J, Barmes D, Beagrie G, Cutress T, Martin J, Sardo-Infirri J. Development of the World Health Organization (WHO) Community Periodontal Index of Treatment Needs (CPITN). Int Dent J. 1982;32:281-91.

12. Michalaki V, Syrigos K, Charles P, Waxman J. Serum levels of IL- 6 and TNF- $\alpha$ correlate with clinicopathological features and patient survival in patients with prostate cancer $\mathrm{Br} \mathrm{J}$ Cancer. 2004;90:2312-6.

13. Sun GW, Shook TL, Kay GL. Inappropriate use of bivariable analysis to screen risk factors for use in multivariable analysis. J Clin Epidemiol. 1996;49:907-16.

14. Sato J, Ohuchi M, Abe K, Satoh T, Abe T, Yamazaki Y, et al. Correlation between salivary interleukin-6 levels and early locoregional recurrence in patients with oral squamous cell carcinoma. Head Neck. 2013;35:889-94.

15. Huang TY, Hsu LP, Wen YH, Huang TT, Chou YF, Lee CF, et al. Predictors of locoregional recurrence in early stage oral cavity cancer with free surgical margins. Oral Oncol. 2010;46:49-55.

16. PoWing Yuen A, Lam KY, Lam LK, Ho CM, Wong A, Chow TL, et al. Prognostic factors of clinically stage I an stage II oral tongue carcinoma-A comparative study of stage, thickness, shape. growth pattern, invasive front malignancy grading, Martinez-Gimeno score, and pathologic features. Head Neck. 2002;24:513-20.
17. Chen Z, Malhotra PS, Thomas GR, Ondrey FG, Duffey DC, Smith CW, et al. Expression of proimflammatory and proangiogenic cytokines in patients with head and neck cancer. Clin Cancer Res. 1999;5:1369-79.

18. Kinoshita A, Tajiri H. Cancer and Inflammation: Suppress Inflammation, Suppress Cancer?. J Transl Med Epidemiol. 2013;1:1004.

19. Roxburgh CS, McMillan DC. Role of systemic inflammatory response in predicting survival in patients with primary operable cancer. Future Oncol. 2010;6:149-63.

20. Czerninski R, Basile JR, Kartin-Gabay T, Laviv A, Barak V. Cytokines and tumor markers in potentially malignant disorders and oral squamous cell carcinoma: a pilot study. Oral Dis. 2014;20:47781.

21. Vučićević Boras V, Čikeš N, Lukač J, Virag M, Cekić-Arambašin A. Salivary and serum interleukin 6 and basic fibroblast growth factor levels in patients with oral squamous cell carcinoma. Minerva Stomatol. 2005;54:569-73.

22. Duffy SA, Taylor JM, Terrell JE, Islam M, Li Y, Fowler KE, et al. Interleukin-6 Predicts Recurrence and Survival Among Head and Neck Cancer Patients. Cancer. 2008;113:750-7.

23. Gümüş P, Nizam N, Lappin DF, Buduneli N. Saliva and serum levels of B-cell activating factors and tumor necrosis factor- $\alpha$ in patients with periodontitis. J Periodontol. 2014;85:270-80.

24. Allen C, Duffy S, Teknos T, Islam M, Chen Z, Albert PS. Nuclear factor-kB-related serum factors as longitudinal biomarkers of response and survival in advanced oropharyngeal carcinoma. Clin Cancer Res. 2007;13:3182-90.

25. Llewellyn CD, Johnson NW, Warnakulasuriya KA. Risk factors for squamous cell carcinoma of the oral cavity in young people-a comprehensive literature review. Oral Oncol. 2001;37:401-18.

26. Woolgar JA. Histopathological prognosticators in oral and oropharyngeal squamous cell carcinoma. Oral Oncol. 2006;42:22939.

27. Linkov F, Lisovich A, Yurkovetsky Z, Marrangoni A, Velikokhatnaya L, Nolen B, et al. Early detection of head and neck cancer: development of a novel screening tool using multiplexed immunobead-based biomarker profiling. Cancer Epidemiol Biomarkers Prev. 2007;16:102-7.

\section{Conflict of interest}

None declared 